\title{
Bildungsexpansion, Reform der Sekundarstufe I und Pädagogische Arbeitsstellen
}

\section{Jürgen Kussau, Lutz Oertel}

Der Beitrag befasst sich mit Funktionen und Wirkungen der Pädagogischen Arbeitsstellen in den Kantonen Bern und Zürich. Sie wurden in den 1970er-Jahren in den Erziehungsdirektionen eingerichtet. Dort sollten sie wissenschaftliche Informationen mit zentraler Planung als neuem Politikmodus verklammern und sich u.a. an einer der Folgen der Bildungsexpansion, der Strukturreform der Sekundarstufe I, bewähren. Die wissensbasierte Verwaltungsreform wurde zwar als Voraussetzung für Schulreform verstanden. Die wissenschaftliche Informationsfunktion geriet jedoch bald in politische Widersprüche zwischen funktionaler Öffnung des Schulsystems (Demokratisierung, Qualifikation) und statusbewahrender Bildungsbegrenzung. Mit dieser Hypothek belastet, konnte Bildungsplanung ihre Handlungsfähigkeit nur in dem Mass sichern, wie sie sich politischen Rationalitätsmassstäben fügte. Zwar lässt sich eine Verwissenschaftlichung der Politik feststellen, wissenschaftliche Informationen bleiben aber in politische Bezüge unterschiedlicher Handlungs- und Wertorientierungen eingebunden.

\section{Einleitung}

Seit den 1950er-Jahren wurde auch die Schweiz vom Prozess der Bildungsexpansion erfasst. Diese nachträgliche begriffliche Verdichtung eines tiefgreifenden gesellschaftlichen Transformationsprozesses wurde in drei Übersetzungsschritten politikfähig gemacht. (1) Auf der Ebene der Ideen und Forderungen kristallisierten sich in der öffentlichen Diskussion als Themen heraus: steigende berufliche Qualifikationsanforderungen, Ausschöpfung eines «Begabtenreservoirs» und Demokratisierung der Bildungsbeteiligung als soziale, regionale, konfessionelle und geschlechtsspezifische Chancengleichheit. (2) Politisch abgearbeitet wurden diese Problemstellungen u.a. über die Umgestaltung der Sekundarstufe I, im Kanton Bern in Verbindung mit der Frage nach der damals noch vierjährigen Dauer der Primarstufe - in der Selbstbeschreibung als "Strukturreform» zu- 
sammengefasst (Hedinger \& Wyttenbach, 1984). Sie beherrschte die schulpolitische Auseinandersetzung in der Schweiz seit den 60er-Jahren bis in die Gegenwart, gekennzeichnet durch eine eigentümliche Ambivalenz zwischen sachlicher Priorität und geringer Relevanz auf der politischen Agenda mit der Folge langwieriger politischer Prozesse bis zum endgültigen Entscheid (Kussau, Oertel \& Wohlgemuth, 1998). Freilich wurde das Thema wellenartig öffentlich brisant und sprengte als gesellschaftspolitischer Konflikt den engen Rahmen der Schulpolitik. Ausserdem überlagerten im Laufe der Jahre immer neue Themen die Strukturfrage und entschärften ihr konfliktives Verteilungspotential. Wichtiger als Schulstrukturen wurden etwa «das Engagement der Lehrer, ihre Ausbildung und ihre Methodik» eingeschätzt (OECD \& EDK, 1989, S. 43); Zürich entschied 1997 über seine Schulstrukturen, als die Autonomiepolitik längst mit höchster Priorität eingeleitet war. Diese auch als Gesamtschulfrage bekannte Diskussion (Bernischer Lehrerverein, 1972; Leschinsky \& Mayer, 1990) wurde schliesslich von einer weiteren Entwicklung begleitet, nämlich (3) der Reorganisation der zentralen Schulbehörden, die in einem engen Zusammenhang mit den genannten Problemstellungen zu sehen ist (Deutscher Bildungsrat, 1974). In die zentralen Bildungsbehörden wurden Planungs- und Informationsfunktionen organisatorisch in Form spezialisierter Abteilungen und durch Rekrutierung wissenschaftlichen Personals eingefügt (Begrifflich bezieht sich "Wissenschaft» hier immer auf die Erziehungs- und Sozialwissenschaften und wird ausserdem auch als Information bezeichnet, um die ambivalente Funktion von Wissenschaft in einem politischen Planungskontext anzudeuten). Die Einrichtung dieser Pädagogischen Arbeitsstellen (im Folgenden Arbeitsstellen; für die deutschsprachige Schweiz: Huberman, 1989) erfolgte im Gegensatz zu materiellen Veränderungen reibungslos.

Mit dem Einbau der Arbeitsstellen erfolgte eine organisatorische Ausdifferenzierung, die informationsbasierte Planung als «neuen Politikmodus» zur «Steuerung» expansiver institutionalisierter Bildungsprozesse etablieren sollte. Die folgenden Überlegungen versuchen, diese Entwicklung zu beschreiben und unter zwei Perspektiven einzuordnen. Zum einen werden materiell die Strukturreformen als Reaktion auf bildungsexpansive Prozesse im Spannungsfeld von Öffnung und Begrenzung des Schulsystems angesiedelt. Zum anderen wird die Einrichtung der Arbeitstellen aus dieser gesellschaftspolitischen Aufgabenstellung heraus in ihrem erwarteten Leistungsbeitrag zur Steigerung der Kapazität der Informationsverarbeitung und «anwendungsorientierten» Überführung in Planung begriffen; die Organisationsreform war selbst Teil dieser Gesellschaftspolitik.

Das Thema der Einrichtung Pädagogischer Arbeitsstellen wird am Beispiel der Kantone Bern und Zürich und ihrer Strukturreformen der Sekundarstufe I behandelt; einmal weil diese Kantone einigermassen untersucht sind, zum anderen weil Bern und Zürch zu den ersten in der deutschsprachigen Schweiz gehörten, die die Vorbereitung wissenschaftlich informierter Schulreformen spezialisierten Organisationsformen übertrugen. Zunächst werden die schulpolitische 
Ausgangslage und die schulstrukturellen Aufgabenstellungen aufgrund der Bildungsexpansion beschrieben. Es folgt eine gesellschaftspolitische Einordnung der Ergänzung der «Politik» durch zentrale «Planung» und des organisatorischen Einbaus der Informations- und Planungsfunktion in die Erziehungsdirektionen, um dann die organisatorische Ausdifferenzierung aufzugreifen. Bevor wir schliesslich in einem kurzen Ausblick den Bogen vom seinerzeitigen Umbau der Zentralbehörden zur gegenwärtig wissenschaftlich und politisch favorisierten einzelschulischen Organisationsentwicklung schlagen, wird theoretisch die Bedeutung und Wirkung der Arbeitsstellen für die Schulpolitik einzuschätzen versucht.

\section{Planung zwischen óffnung und Begrenzung}

Die schulpolitische Konzentration auf die Strukturfrage kann als Folge der Bildungsexpansion verstanden werden, weil Schulstrukturen die Verteilung von Qualifikationen und gesellschaftlichem Status vermitteln und umgekehrt expansive (und kontraktive) Entwicklungen diesen Verteilungsmechanismus unmittelbar tangieren. Charakterisiert war die Expansionsentwicklung durch die Merkmale «mehr, grösser, länger und differenzierter» - insgesamt durch einen zunehmenden «Verschulungsgrad» der Gesellschaft (Bornschier \& Aebi, 1992, S. 559 f.).

- Es wurde ein «mehr» an Bildung nachgefragt. Die Bildungsansprüche erweiterten sich auf höhere Abschlüsse;

- das Bildungssystem erfasste grössere Bevölkerungskreise in weiterführenden Bildungsinstitutionen ab der Sekundarstufe I (Ausweitung der Bildungsbeteiligung);

- die Verweildauer in Bildungsinstitutionen verlängerte sich;

- schliesslich wurde das schulische Angebot zunehmend differenziert.

Formal stellte sich also der Schulpolitik die Aufgabe, einen «genügenden Primarunterricht» ${ }^{1}$ als staatliches Angebot zu sichern, zu organisieren und weiterzuentwickeln. Insofern ist das politische Bildungskonzept dieser Zeit als «quantitativ» zu bezeichnen (Stricker, 1991, S. 1). Die zwei- oder dreigliedrige Sekundarstufe I war durch die Verschiebungen in der Verteilung der Schülerinnen und Schüler zwischen den Stufenangeboten auf der Sekundarstufe I hin zu den anspruchsvolleren Abschlüssen aufgrund der steigenden Qualifikationsanforderungen rein quantitativ nicht mehr gewachsen. Deshalb kam es zum Beispiel in Bern zur «explosionsartige Vermehrung der Sekundarschulen» (Stricker, 1991, S. 1; Rogger, 1992, S. 420 f.). Im quantitativen Ausdehnungsprozess prägte sich der Mechanismus des «sozialen Aufstiegs durch Bildung» aus (Lutz, 1983, S. 223), der seinerseits wieder zirkulär die Bildungsexpansion vorantrieb. 
Im Gefolge von Industrialisierung und Modernisierungsprozessen ${ }^{2}$ veränderte sich die Gesellschaft nachhaltig: Entwicklung der «Dienstleistungsgesellschaft» mit Verschiebungen zwischen primärem, sekundärem und tertiärem Sektor (für Zürich: Erziehungsdirektion, 1990) ${ }^{3}$, entsprechenden soziostrukturellen Auswirkungen und geänderten Bildungsanforderungen. Der Anspruch breiter(er) Bevölkerungskreise auf Teilhabe an Bildung erweiterte sich über die im 19. Jahrhundert etablierte Elementarbildung hinaus auf weiterführende Bildungseinrichtungen und erfasste nicht nur das Gymnasium und die Hochschule, sondern auch die Sekundarstufe I. In diesem Sinne kann man die Bildungsexpansion im Prozess «sozialer Demokratisierung» (Bornschier \& Aebi, 1992, S. 548) als nicht intendierte Folge der Verallgemeinerung der Volksschule im 19. Jahrhundert begreifen (Schneider, 1982). Die nach langen Auseinandersetzungen etablierte "Schule für die Demokratie» schloss aus (Criblez et. al. 1999, Jenzer, 1997), Bildung als zunehmend verwertungsbezogene Bildungsansprüche zu begrenzen. Einer umstandslosen Fortsetzung der Bildungsexpansion als einer das Bildungssystem erst legitimierenden «politischen» Öffnung des Schulsystems stand freilich die schulische Funktion entgegen, als wesentliche «soziale Dirigierungsstelle» der Zuteilung von «Lebenschancen» zu wirken (Schelsky, 1957, S. 17 f.). Besonders in stratifizierten Schulsystemen wie in denen der Schweiz leisten die Schulen einen massgeblichen Beitrag zur späteren beruflichen Positions- und gesellschaftlichen Statuszuweisung (Allmendinger, 1989; Buchmann \& Sacchi, 1998). Verknappung des öffentlichen Guts Bildung und «Statuskonkurrenz» liessen eine aktiv-expansive Bildungspolitik nicht zu (Bornschier \& Aebi, 1992, S. 554 ff.), weil der jeweils erreichte Bildungs- und Sozialstatus mit der Folge von Verteilungskonflikten verteidigt wurde.

Während die Zeichen einerseits auf Ausweitung des Bildungssystems gerichtet waren, «mussten» aus gesellschaftspolitischen Gründen gleichzeitig zertifikationsbezogene Zugangsbeschränkungen zu weiterführender Bildung aufrechterhalten werden, da das Bildungssystem als Legitimationsinstanz sozialer Ungleichheit über leistungsbezogene Selektionsprozesse in mehr oder weniger stark differenzierten Strukturen fungiert (Bornschier \& Aebi, 1992; Graf \& Lamprecht, 1991). Der Schulpolitik wurde ein Spagat zwischen Ausschöpfung der Bildungsreserven und Demokratisierung der Bildungszugänge bei gleichzeitiger Bildungsbegrenzung abverlangt, der alle kommenden Strukturdiskussionen be-stimmte. ${ }^{4}$ Für die Bildungs- und speziell die Schulpolitik ergab sich daraus die Notwendigkeit, den eigendynamischen Prozess der Bildungsexpansion als Konkurrenz zwischen Statusverbesserung und -erhaltung (Bornschier \& Aebi, 1992, S. 551 ff.) und die eher naturwüchsige Beziehung von Bildungsund Beschäftigungssystem im Dreieck von Bildungsangebot, -nachfrage und qualifikatorischem Bedarf systematisch(er) zu kanalisieren und aufeinander abzustimmen.

Diese langfristigen Entwicklungsverläufe, die in Prozesse der «Dynamisierung» und des "Aufbruchs» der Schweiz in den 60er- und 70er-Jahren eingebet- 
tet waren (König et al., 1998), spitzten sich in den 60er-Jahren durch spezifische Ereignisse zu. Besonders der «Sputnik»-Schock ist hier zu erwähnen und die daraufhin einsetzende Krisendiagnose in den westlichen Ländern. "Bildungsnotstand» (Rogger, 1992, S. 397 ff.) oder auch «Bildungskatastrophe» (Picht, 1965) beschrieben konsensfähig den Zustand des Bildungssystems. «Bildung» als «Bürgerrecht» (Dahrendorf, 1968) verwies auf Reformerwartungen und vermutete -potentiale der Bildungs- und Schulpolitik, die weit über ihre Qualifikationsfunktion im Dienste wirtschaftlichen Wachstums hinausreichten und besonders der Schule die Rolle einer «Stellgrösse im System sozialer Ungleichheit» zudachten (Baumert, 1991, S. 333; SKBF, 1998, S. 61 ff.).

Der qualitative Zusammenhang sowohl zwischen Bildung und Beschäftigung als auch zwischen Bildung und Demokratisierung leiten sich ab von den Erwartungen an die Schule, (u.a.) als Ort des Erwerbs von und der Einübung in universalistische Handlungsorientierungen in der zivilisatorischen Entwicklung der Gesellschaft zu fungieren. Deshalb kann es nicht überraschen, dass das Bildungsund Schulsystem im Prozess der Entwicklung von Staatsbürgerrechten über bürgerliche und politische Rechte hinausdrängte und auf soziale Rechte zielte. Die Formel von «Bildung ist Bürgerrecht» (Dahrendorf, 1968) richtete sich auf sozialen Aufstieg durch Bildung und liess soziale Ungleichheit qua autoritativer Zuweisung entlang sozialer Herkunft und geschlechtsspezifischer, konfessioneller und regionaler Unterschiede nicht nur normativ als defizitär, sondern auch als vormodern und disfunktional erscheinen. «Aktive Bildungspolitik» (Dahrendorf, 1968) im Sinne einer Demokratisierung der Bildungssysteme, programmatisch formuliert als Chancengleichheit, erschien geboten und sollte gezielt zur «Expansion des Bildungswesens» und der Modernisierung der Gesellschaft eingesetzt werden (Rogger, 1992, S. 397 ff.; SKBF, 1996, S. 64 f.).

In der Wachstumsphase vor Beginn der Rezession 1973/74 bestanden Verteilungsspielräume, so dass in historischer Koinzidenz ökonomisch geforderte Ausschöpfung von Begabungsreserven und Demokratisierung als Öffnung des Schulsystems miteinander koexistierten und sich wechselseitig vorantreiben konnten. In der Wirkung, nicht in den Absichten, waren beide Ansprüche austauschbar. Erst mit Beginn der Rezession prägte sich die tiefgreifende Differenz als Verteilungskonflikt um berufliche Positionen und sozialen Status aus und auch für die Strukturveränderungen in Bern und Zürich gilt: «Was bis dahin an Neuerungen nicht durchgesetzt war, hatte [...] mit verschärften Widerständen zu rechnen» (König et al., 1998, S. 11). Beide Kantone kamen in dieser Hinsicht «zu spät». In Bern gelang 1972 nur noch ein einzelner Schulversuch («Manuelversuch»; Lüscher, 1995, S. 47 ff.) - dem später weitere folgten -, nicht hingegen eine umfassende Revision der Bildungsgesetzgebung. Und Zürich geriet mit seinen Strukturreformabsichten ab 1976 (Oertel, 1997, S. 81 ff.) bereits in die rezessive Wirtschaftsphase. Einzig der Kanton Tessin konnte die Gunst der Stunde nutzen, indem er bereits 1972 die gesetzlichen Grundlagen für die Einführung der Scuola Media schuf (Kussau, Oertel und Wohlgemuth, 1998). 
Die hier grob umrissene Problemlage wurde in ihrer gesteigerten Komplexität jedenfalls als Aufgabe, u.a. die Schulstrukturen auf der Sekundarstufe I zu bearbeiten, registriert (nicht als Bildungsexpansion). In ihrem Gefolge stellte sich die Frage nach den dafür geeigneten politisch-administrativen Kapazitäten. Das «Gespür» für administrativ-konzeptionelle Defizite, mit dieser Aufgabe angemessen umzugehen, verdichtete sich zu einer funktionalen Notwendigkeit. Verwaltungsreform geriet zur Voraussetzung einer Schulreform. Damit wird kein deterministischer Zusammenhang angenommen. Sowohl organisatorische Varianten wie Ungleichzeitigkeit organisatorischer Ausdifferenzierungsprozesse Basel-Stadt verzichtete auf die Einrichtung einer Arbeitsstelle, viele Kantone richteten sie erst später ein - deuten auf kontingente Prozesse hin. (Unterschiedlich) wahrgenommene Modernisierungsnotwendigkeiten, Prozesse der Verwissenschaftlichung und gewandelte kollektive Deutungsmuster von Problemen und Politikangeboten legten jedoch in Bern und Zürich in Verbindung mit einem zentralen «Planungsanspruch» die Steigerung institutioneller Politikfähigkeit qua Verwaltungsreform nahe.

Aufgaben und Leistungsfähigkeit der Arbeitsstellen waren jedoch durch die angedeuteten Widersprüche zwischen demokratisierender und qualifikatorischer Öffnung des Schulsystems (integrative und kooperative Schulmodelle) - aktiv die Schule als «Stellgrösse» begreifend - und statusbewahrender Bildungsbegrenzung mit einer Hypothek belastet. Während die Arbeitsstellen sich dem Öffnungsgedanken verschrieben hatten, zunächst vor allem lokale Strukturprobleme evident waren und wissenschaftlich vielfach Reformnotwendigkeiten angenommen wurden, waren dafür über Jahre hinweg weder in Bern noch in Zürich politische Mehrheiten vorhanden (Entscheide in Bern 1991 und 1997 in Zürich), und auch die aktive Unterstützung durch die jeweiligen Erziehungsdirektoren war, von Ausnahmen abgesehen, kaum vorbehaltlos gegeben. Wie die Geschichte der Strukturreformen in beiden Kantonen zeigt, bestand kein linearer und schon gar nicht ein determinierender Zusammenhang zwischen gesellschaftlichen Veränderungen und Anforderungen an das Bildungssystem und ihrer politischen Umsetzung. Ob ein solcher Zusammenhang überhaupt besteht, war (und ist) vielmehr Ergebnis politischer Registrierung eines Problems in je spezifischen gesellschaftlichen Konstellationen und daraus folgenden Machtauseinandersetzungen. In Bern und Zürich bot Schulpolitik für eine strukturkonservative Mehrheit den politischen Ort des «Widerstands» - Schule als «soziale Dirigierungsstelle» und Hort kultureller Tradierung.

Der Auftrag an die wissenschaftlich orientierten Arbeitsstellen war mithin höchst ambivalent. Gleichzeitig sollten die schulstrukturellen Folgen der Bildungsexpansion in ihrem Problemgehalt bearbeitet, Qualifikationsniveaus gehoben («keine Niveausenkung») und die gegebene pyramidale Positions- und Statusverteilung nicht tangiert werden, so dass an Gleichheitsvorstellungen ausgerichtete Demokratisierungsforderungen zur Veränderung der Reproduktion der Strukturen der Statuszuteilung sich nicht einstellten (Lam- 
precht/Stamm 1996). Als wissenschaftlich orientierte Planungsabteilungen waren die Arbeitsstellen in diesen widersprüchlichen Zusammenhang eingewoben. Sie sollten ihn durch umfassende Konzepte aktiv und antizipativ «auflösen» - zukunftssicherer machen - und zugleich gesellschaftliche Strukturmuster bewahren. Angesichts dieser über die Schulpolitik hinausreichenden Politisierung waren wissenschaftlichen "Objektivierungspotentialen» in der Strukturfrage von Anfang an Grenzen gesetzt: Sie zeigten sich, als die Arbeitsstellen versuchten, den Planungsauftrag auch «inhaltlich» auszuformulieren, indem sie advokatorisch den bisher nicht berücksichtigten und nur schwer organisierbaren Interessenstandpunkt der schulischen Demokratisierung aufnahmen («advocacy planning») und in kooperative/integrative, durchlässige Schulmodelle, veränderte Selektionsmechanismen und Unterrichtsformen übersetzten, die sich an Prinzipien der Individualisierung und Differenzierung ausrichteten.

Bildungsexpansion und damit die Ausgangslage der Arbeitsstellen können als historische Überraschung beschrieben werden. In Zürich etwa hatte man erst 1959 eine dreigliedrige Sekundarstufe I eingeführt (Wymann, 1993, S. 63 ff.), ohne die sozioökonomische Transformationsdynamik zu berücksichtigen, so dass die Politik bereits zehn Jahre später von der expansiven schulexogenen Entwicklung eingeholt wurde, die die gerade geschaffenen Schulstrukturen wieder in Frage stellte. Schul- und Bildungsplanung erwiesen sich deshalb auch weniger als aktive Reformpolitik - diese Charakterisierung trifft nur auf die Arbeitsstellen selbst zu - denn als Reaktion auf unabweisbare Problemlagen. Jenseits einer gesellschaftlichen und politischen Gesamtsicht waren die Anlässe schulstruktureller Veränderungen «greifbarer». Politische Aktivitäten wurden ausgelöst durch konkrete, lokale - nicht kantonsweite - Funktionsdefizite und Bestandsgefährdungen einzelner Schulhäuser bzw. Schultypen. Ortsspezifische, demographische Veränderungen sind als Ursachen ebenso zu nennen wie die Entwicklung von «Restschulen» im Zuge steigender Bildungsansprüche (Oertel, 1997, S. 67 f.) oder umgekehrt das fehlende Angebot von weiterführenden Schultypen bei gleichzeitig vorhandener Nachfrage (Anlass für die Schulversuche in Twann und Schangnau im Kanton Bern; Kussau, 1998). Hier waren unmittelbar die lokalen und zentralen Schulbehörden gefordert, woraus interaktive Formen «zentral-lokaler» Problembearbeitung resultierten. Diese Ausgangslage führte im $\mathrm{Zu}$ sammenspiel mit dem ambivalenten Auftrag dazu, sich vorsichtig über Schulversuche an die Aufgabe heranzutasten. Im Kanton Bern bildete sich, nachdem eine Gesamtrevison der Bildungsgesetzgebung keine Mehrheit gefunden hatte, ein Schulversuchstypus heraus, der lokale Sonderprobleme für kooperative und integrative Strukturmodelle zur gleichzeitigen Problemlösung nutzte, während der Kanton Zürich lokale Probleme als kantonsweite Strukturprobleme identifizierte und daraus die Strategie einer systematischen Versuchsanlage ableitete. 


\section{«Entdeckung» politischer Planung im Bildungs- system}

Charakteristisch für den damaligen Politikansatz war funktional der Einbau der Informations- und Planungsfunktion in die zentralen Erziehungsdirektionen, um die «Steuerungskapazität» des politisch-administrativen Systems zu erhöhen. Schulische Veränderungsprozesse sollten zentral (kantonal) geplant werden 5 (wobei Strukturveränderungen aus konstitutionellen Gründen zentral angelegt sein mussten). Denn «aktive» Bildungspolitik zur Gesellschafts- und Institutionengestaltung war in den 60er- und 70er-Jahren noch eingebettet in ein wohlfahrtsstaatliches Verständnis politischen Handelns, wonach "durch den Staat oder staatliche Regelungen dazu beigetragen werde, mit Hilfe von Schulen bestimmte Ziele und Erwartungen zu erreichen» (Ekholm, 1997, S. 597; SKBF, 1996, S. 62 ff.; de Swaan, 1993). Die Erweiterung der Erziehungsdirektionen um Planungskapazitäten war selbst Ausdruck dieses zentralen Planungsansatzes und stand im Kontext einer quantitativen und qualitativen Ausweitung der Staatsaufgaben bzw. des öffentlichen Sektors (Weltbank, 1997).

Die bildungsexpansive Ausgangslage und ihre Folgen für die politische Gestaltung des Schulsystems legten eine stärkere informatorische Fundierung der Politik nahe. Politik «von Hand» genügte nicht mehr. ${ }^{6}$ Die «alte» Politik, die auf Routinen, "Verwaltung» der Schule, institutionellen Formen und Teilveränderungen beruhte, wurde mit den komplexeren Anforderungen nicht mehr fertig. Die Tertiärisierung der Gesellschaft, ihre sozialstrukturellen Verschiebungen und die daraus erwachsenden individuellen Bildungskompetenzen und darauf gerichteten Bildungserwartungen und -entscheidungen «erforderten» informationsgestützte Planung und plausible Begründungen für politische Interventionen. Im Zuge der Rationalisierung und Verwissenschaftlichung der Gesellschaft bekam informationsbasierte Planung grössere Bedeutung als Ergänzung oder sogar als Ersatz «älterer» Gestaltungsformen.

Information wurde dabei verstanden als wissenschaftlich fundierte, empirische Datensammlung und Verknüpfung dieser Daten zu Ursache-WirkungsKetten mit dem Ziel einer Identifizierung politisch beeinflussbarer Faktoren. Dezidierten Ausdruck fand dieses analytische Informationsverständnis im Aufbau systematischer gesellschaftlicher Beobachtungsformen («Monitoring») auf der Grundlage sozialer Indikatoren. Wesentliche Eigenschaften der optimistischen, damals noch nicht enttäuschten, sozialtechnologischen «Logik» informierter Planung waren aufgehoben in Konzepten wie «aktiver» ${ }^{6}$ anstelle «reaktiver» Politik (Mayntz \& Scharpf, 1973) oder politischer "Gesellschaftsplanung». Für Bildung schlugen sie sich nieder in Ideen einer «aktiven Bildungspolitik» (Dahrendorf, 1968) und "Bildungsplanung als rollende[r] Reform» (Rolff, 1970) - in einem breiten Variantenspektrum und von Beginn an auch kritisch gedacht bzw. begleitet (Linder, Hotz \& Werder, 1979; allgemein Offe, 1972a; Naschold, 1974). Dieses Verständnis politischer Planung lässt sich als 
Steigerungsform politisch-administrativer Interventionsfähigkeit zusammenfassen. Weniger abstrakt formuliert sahen sich Industriestaaten nun in der Lage, gesellschaftliche Funktionssysteme auf professioneller Grundlage unter Einsatz von Wissen(schaft) zu gestalten (Moynihan, 1965/66, S. 10). Die Idee wurde getragen durch den "Glauben» an die Machbarkeit gesellschaftlicher Verhältnisse («Fortschritt») auf der Basis der (sachlichen) Analyse realer Gegebenheiten, expliziter Zielorientierung der Politik, Entwurf alternativer Handlungspfade und in zeitlicher Hinsicht Ausprägung antizipativer Kapazitäten gestützt auf (sozial)wissenschaftliche Prognosefähigkeiten und dadurch Erschliessung einer grösseren Optionsvielfalt und längerfristigerer Zeithorizonte. Neben «objektive» Planungsnotwendigkeiten - und damit verschränkt - traten also gesellschaftspolitische «Vorstellungen» einer «umfassenden sozialen Planung und Steuerung». Was für den «Konjunkturdiskurs» festgestellt wurde, trifft auch für den Bildungsdiskurs zu. Er «entfaltete sich in Richtung eines zunehmend technokratisch verstandenen Glaubens an die Kontrollierbarkeit sozialer Prozesse» (König et al., 1998, S. 13). Planung besetzte genau die Schnittstelle zwischen gesellschaftlichen Erfordernissen und - auch durch Verwissenschaftlichung erzeugter - politischer Intentionalität. Dazu bedurfte es organisatorischer Anpassungsleistungen.

Aktive Politik qua Planung gehört in den theoretischen Zusammenhang von politisch intendierter Gesellschafts- und Institutionengestaltung (Czada \& Schimank, 2000; Goodin, 1996). Während in politikpraktischer Hinsicht Intentionalität "offenbar» alternativlos «zu den rationalen Grundlagen politischen Handelns» gehört (Czada \& Schimank, 2000, S. 25) und im Theoriestrang demokratischer Konsens- und Deliberationskonzepte abgedeckt ist, werden Veränderungsprozesse theoretisch auch alternativ erklärt, sei es über selbstregulative Marktprozesse, sei es über evolutive oder kontingente Entwicklungen. Jedenfalls sind emergente Institutionen- und Gesellschaftsdynamiken oder eigendynamische Prozesse, die, einmal ausgelöst, sich «aus sich selbst heraus und ohne weitere externe Einwirkung weiterbewegen», das Komplement intendierter Gestaltung. Emergente Dynamiken entziehen sich Intentionalität und werden zum "Gegenbegriff zu 'Plan- und Steuerbarkeit'» (Zitate Mayntz \& Nedelmann, 1987, S. 648 f., S. 651), sind aber gleichzeitig auch mit intentionaler Politik verbunden, denn selbst gelingende Intentionalität erzeugt eigengesetzliche (nicht intendierte) Dynamiken. Die theoretischen Zugänge unterscheiden dezidiert die Bedeutung politischer Intentionen für die Gestaltung der Gesellschaft. Evolutions- und Markttheorien relativieren politisch intendierte Programme, wenn sie sie nicht sogar als «kleinzeitige» Episoden in Frage stellen (Fuchs, 1999, S. 157) oder als «Staatsversagen» kennzeichnen (Wolf, 1993). Deshalb «passen» sie nicht für intentionale Politik, will sie ihre legitimen Gestaltungsansprüche und -erwartungen nicht aufgeben. Theorien, die politische Intentionen berücksichtigen, gehen deshalb von (relativ) autonomen politischen Steuerungsleistungen aus (Nordlinger, 1981), ohne auszuschliessen, dass die Politik von exogenen Pro- 
zessen getrieben wird und materielle Politikprogramme (policy) das Ergebnis vielfach interferierender Einflüsse politischer (staatlicher) und gesellschaftlicher Akteure sind mit fliessenden Übergängen zwischen intentionaler Gestaltung und «dynamischer Emergenz» (Czada \& Schimank, 2000, S. 24).

Um diese Spannung zwischen autonomer Politikgestaltung und exogener Abhängigkeit kreisen auch analytische Einschätzungen der politisch induzierten Strukturveränderungen der Sekundarstufe I. Waren sie im Zuge der Bildungsexpansion Akte «bewussten und geplanten gesellschaftlichen Fortschritts» oder «notwendig, um einen volkswirtschaftlichen Bedarf an höherer Qualifikation zu decken» (Lutz, 1979, S. 647)? Die Arbeitsstellen in Bern und Zürich siedelten sich in ihren Handlungsorientierungen auf der intentionalen Gestaltungsseite an. Davon zu unterscheiden sind die Absichten der politischen Institutionen, die die Einrichtung der Arbeitsstellen bewilligten. Ihre Aufgabenzuschreibung war auf die Etablierung wissenschaftlicher Kapazitäten in «gestalterischer Absicht an sich» gerichtet, nicht auf ihren Beitrag zu Strukturveränderungen (siehe oben). Entsprechend können die Arbeitsstellen als organisatorische Ausprägung der gebotenen Planungs- und Informationsfunktion im Zuge der wahrgenommenen Aufgabenveränderungen verstanden werden. Eingefügt in die Verwaltung werden sie Teil des «arbeitenden Staates», der «hierarchisch-integrativ» gedachten, «vorgeordneten» Institution zur Einwirkung der Gesellschaft auf sich selbst. Die binnenorganisatorische Erweiterung der zentralen Bildungsbehörden um Planungskapazitäten löste, wie die Aktivitäten der Arbeitsstellen zeigen, das Verständnis der Erziehungsdirektionen als «neutralen Exekutivbehörden» auf und ergänzte sie über routinisiertes «Schulmanagement» hinaus in Richtung "planender Verwaltung» - formal wissenschaftlich orientiert, materiell diffus. Wie es im Kanton Zürich in der Aufgabenbeschreibung der Pädagogischen Abteilung (PA) hiess:

- Der «Rhythmus», in dem neue Anforderungen «an die Schule herangetragen werden», hat sich "wesentlich beschleunigt».

- Weiter sieht sich die Schule vermehrt «widerstreitenden Anforderungen» gegenüber.

- Und schliesslich wird auf die «strukturellen Verflechtungen» verwiesen, die isolierte Massnahmen "ohne Beeinflussung des gesamten Schul- und Bildungswesens» undenkbar werden lassen (PA ZH, 1974, S. 523; Hervorhebung im Original).

Bildungsplanung sollte die Funktionsstelle der "planenden Verwaltung» besetzen, um in Form von "Reformpolitik» Voraussetzungen und Folgen von politischen Programmen konzipieren zu können. Information und Planung wurden zu Synonymen für die möglichst genaue Bestimmung notwendiger und möglicher Veränderungen, nicht zuletzt, um sie unter Bedingungen von Ressourcenknappheit politisch «so eng wie möglich begrenzen zu können» (v. Friedeburg \& Oehler, 
1984, S. 257). Und eine zentrale Bestimmungsgrösse waren die Schulstrukturen. Anders als heute mit dem «outsourcing» staatlicher Aufgaben vielfach propagiert, richteten sich die damaligen Aktivitäten auf strukturelle und funktionale Ausdifferenzierung der Erziehungsdirektionen selbst, Steigerung ihrer «Informations- und Problemverarbeitungskapazität», sowie Professionalisierung und Verwissenschaftlichung der Aufgabenbearbeitung. Im zirkulären Prozess der Verwissenschaftlichung der Politik nahm ausserdem der Begründungsbedarf für Politik zu, der wieder eine informationelle Grundlegung politischer Massnahmen vorantrieb. Den gesellschaftlichen Modernisierungsprozessen musste die Rationalisierung der Politik folgen oder diese erst vorantreiben (Lutz, 1979, S. 647 f.). Entsprechende Aktivitäten richteten sich ausschliesslich auf die "Exekutive», nicht die «Legislative». Die Stärkung der Zentralbehörde ging einher mit einer Vergrösserung des Informationsungleichgewichts zwischen Regierung und dort vor allem der Verwaltung und den gesetzgebenden Institutionen sowie dem Milizsystem.

Wenn man Politik analytisch differenziert in materielle Programme («policy») zur «Lösung» von Problemen oder sogar zur «Zukunftsgestaltung», die in institutionell geregelten Auseinandersetzungen um Mehrheiten und Machtanteile («politics») entschieden werden, dann setzen Versuche politischer Rationalisierung über den Einbau einer verwissenschaftlichten Informationsfunktion auf der «Policy-Seite» mit dem Ziel an, die politischen Prozesse («Politics-Seite») zu versachlichen: Mehrheitsbeschaffung qua begründeter und gleichsam unbestreitbarer (selbstevidenter) Informationen über gesellschaftliche (schulische) Zustände und ihre kausalen Zusammenhänge als Grundlage gezielter Veränderung. In einer pluralistischen und komplexeren Gesellschaft mit gesteigerter Interdependenz von Akteuren, Ereignissen, Situationen, Handlungen, funktionalen Teilsystemen und mit potentiell konfliktiven Politikprozessen, die überhaupt erst die Notwendigkeit kollektiven politischen Handelns begründen, schienen sich unter konstanten institutionellen Bedingungen auf der «Politics-Seite» unüberwindbare Hürden politischer Rationalisierung aufzutun. Planung konnte hingegen als Rationalisierung materieller Politik («Policy-Seite») Eingrenzung der «Willkür» von «Wahlakten» verheissen (Luhmann, 1993, S. 287 ff.) und Politik als «informierte» Politik ausweisen. Selbstevidente empirische Fakten zusammengeführt in politischen Programmen (policy) hätten Ergänzung, wenn nicht sogar Ersatz von "common sense» sein und politische Konflikte «objektivieren» und der politischen Auseinandersetzung «entziehen» sollen - in Direktdemokratien ein systemwidersprüchlicher Ansatz. «From causation to decision: planning as politics» (Cohen, 1970) beschreibt (kritisch) das Programm in seinen tragenden Komponenten und auch seiner Widersprüchlichkeit, die in der Technokratiethese kulminiert, in der «sachgesetzliche» Planung an «die Stelle eines politischen Volkswillens tritt» (Schelsky, 1979, S. 465). 


\section{Die Einrichtung Pädagogischer Arbeitsstellen}

Wenn man an Organisationen Fragen nach ihrer Grösse und Struktur, ihrer personellen und sachlichen Ausstattung, ihren Aufgaben, ihrer Kompetenz, ihrer Handlungsfähigkeit und ihrer Legitimität - verstanden als Selbstlegitimierung im Sinne binnenorganisatorischer Akzeptanz - stellt, so liegt das Schwergewicht im Folgenden, neben einer Aufgabenbeschreibung, auf den beiden letztgenannten Themen.

\section{Aufgaben und Arbeitsschwerpunkte der Pädagogischen Arbeitsstellen}

Charakteristisches Merkmal des Aufgabenzuschnitts der Arbeitsstellen in Bern und Zürich war die "Gesamtüberprüfung des Unterrichtswesens aller Stufen» (PA ZH, 1972, S. 1) im Sinne einer Bestandsaufnahme des Bildungssystems als «Einheit» (PA ZH, 1972, S. 2). Gedacht war sie als Grundlage der «mittel- und langfristigen Planung im Bildungswesen» (PA ZH, 1972, S. 1; Hervorhebung im Original) durch die neuen organisatorischen Einheiten. Zwischen materieller Funktion und organisatorischer Ausdifferenzierung wurde ein unmittelbarer Zusammenhang hergestellt. Reformpolitik bedurfte als Voraussetzung der «Organisationspolitik» in Form einer Reorganisation und Modernisierung der Erziehungsdirektionen. Die "Verwaltungsarbeit der Erziehungsdirektion» (im Kanton Bern) konnte «bis in die letzte Zeit hinein mit einem relativ geringen personellen Bestand bewältigt werden; das Gesetzgeberische erfolgte durch die Spitze der Direktion zusammen mit den Inspektoren; die Überwachung der Schularbeit durch die Inspektoren stellte bis in die Fünfzigerjahre wegen der geringen Rotation der Lehrerschaft keine ernsthaften Probleme» ([Erziehungsdirektor] Kohler, 1973, S. 1). Diese Funktionsweise genügte nicht mehr. Als Mangelsituation wurde sie aber nicht durch konventionelle Aufstockung von Personal und auch nicht durch Intensivierung milizförmiger Zuarbeit zu beheben versucht, sondern durch Einfügung der spezialisierten Planungs- und Informationsfunktion in die Erziehungsdirektionen und entsprechende Professionalisierung und Verwissenschaftlichung der Aufgabenerledigung (Steigerung der «Informationsverarbeitungskapazität»; Ergänzung individuell-serieller durch kollektiv-parallele Informationsverarbeitung).

Den Arbeitsstellen in Bern und Zürich wurden folgende Aufgaben zugewiesen: 
Aufgaben der Pädagogischen Arbeitsstellen in Bern und Zürich (Bern-Dekret, 1971, Art. 15; PA ZH 1972, S. 3 ff.)

\begin{tabular}{|c|c|}
\hline $\begin{array}{l}\text { Kanton Bern } \\
\text { Amt für Unterrichtsforschung (AfU) }\end{array}$ & $\begin{array}{l}\text { Kanton Zürich } \\
\text { Pädagogische Abteilung (PA) }\end{array}$ \\
\hline $\begin{array}{l}\text { - "Beratung der Direktion in erziehungs- und } \\
\text { bildungswissenschaftlichen Fragen»; } \\
\text { - "Sammlung wissenschaftlicher Erkenntnisse», } \\
\text { ihre Vermittlung sowie Umsetzung zur "prak- } \\
\text { tische(n) Anwendung»; } \\
\text { - "Verbindung zu wissenschaftlichen Institutio- } \\
\text { nen und zur Schulpraxis»; } \\
\text { - "Anregung von Untersuchungen und Versu- } \\
\text { chen, gegebenenfalls deren Durchführung [...] } \\
\text { und Auswertung»; } \\
\text { - "Mitarbeit in allen Fragen der Aus-, Fort- und } \\
\text { Weiterbildung». }\end{array}$ & $\begin{array}{l}\text { - } \text { "Erarbeitung von Planungsgrundlagen (Kon- } \\
\text { zeptionen)» als "Bearbeitung von pädagogi- } \\
\text { schen Problemen innerhalb der Verwaltung» } \\
\text { und Durchführung bzw. Planung "spezieller } \\
\text { Untersuchungen und Erhebungen». } \\
\text { - Kantonale und interkantonale Koordination } \\
\text { der "pädagogischen Bestrebungen»; } \\
\text { - Dokumentations- und Informationsverarbei- } \\
\text { tung als «Informationssammlung» und -ver- } \\
\text { mittlung an die (bildungs-)politischen Institu- } \\
\text { tionen; } \\
\text { - Bildungsstatistik: neben allgemeinen Optimie- } \\
\text { rungen der Bildungsstatistik ist die Rede von } \\
\text { "Planung eines kantonalen Schulinformations- } \\
\text { systems» und von "Prognosen [...] über die vo- } \\
\text { raussichtliche Entwicklung von Schülerzahlen» } \\
\text { sowie von der Erhebung demographischer Da- } \\
\text { ten. }\end{array}$ \\
\hline
\end{tabular}

Das Aufgabenspektrum der Planungseinheiten war gegenüber der Politik als Entscheidungssystem auf Beratung, wissenschaftliche Untersuchungen, Vermittlungsleistungen, kurz: Politikvorbereitung festgelegt. Materiell lässt sich ein spezifisches Verständnis von Bildungsplanung erkennen, indem es dezidiert auf «bildungswissenschaftliche» Erkenntnis und Umsetzung bzw. auf "pädagogische» Themen begrenzt war. Das Wechselverhältnis zwischen Bildungssystem und Gesellschaft sollte nur unmittelbar schulbezogen erfasst werden. Das systematische Ungleichgewicht zwischen Überqualifikation und Unterqualifikation, die Abstimmung zwischen qualifikatorischem Schulangebot und Qualifikationsnachfrage und der Zusammenhang zwischen Lehrstellenangebot und Konjunkturverlauf waren nicht in der Aufgabenbeschreibung enthalten. Zwar wurde Schulpoltik als Wirtschafts- und Beschäftigungspolitik begriffen. Daraus wurden aber nur begrenzt - jedenfalls in den Arbeitsstellen - bildungsplanerische Konsequenzen gezogen, wenn darunter verstanden wird, Bildungssystem und Beschäftigungssystem aufeinander abzustimmen. Damals ging es zunächst darum, das Qualifikationsniveau des öffentlichen Schulangebots in Reaktion auf steigende Bildungsansprüche als individuelle und kollektive Bildungspräferenzen zu steigern. In den 60er- und 70er-Jahren hatten sich Bildungssystem und -politik «relativ» verselbständigt («relative Autonomie»; Baethge \& Teichler, 1984), vor allem auch gegenüber anderen Politiksektoren wie Wirtschafts- und Sozialpolitik, so dass begrenzte (volks)schulbezogene Bildungsplanung als Gestaltung von Angebotsveränderungen zwischen den Schultypen vor allem auf der Sekundarstufe I praktiziert werden konnte. 
Dieser «engere», bildungswissenschaftliche und pädagogische Aufgabenschwerpunkt kommt in der Bezeichnung der Arbeitsstelle in Bern als "Amt für Unterrichtsforschung und -planung» (AfU) und in Zürich als "Pädagogische Abteilung» (PA) treffend zum Ausdruck. Der unmittelbar und nur auf die (Volks)Schule in ihrer Kernkompetenz bezogene Politikansatz sollte beibehalten werden und keine Anlehnung an «umfassende Bildungsplanung» gesucht werden. Der Kanton Bern hat mittlerweile in der neuen Bezeichnung des AfU als "Amt für Bildungsforschung» begrifflich die Perspektive ebenso geweitet wie der Kanton Zürich, der die PA in Abteilung für «Bildungsplanung» umwidmete.

Bildungsplanung spielte in den 70er- und 80er-Jahren als umfassende Aufgabe in den Arbeitsstellen nur in eingeschränkter Form eine Rolle. Auch eine Untersuchung in der deutschsprachigen Schweiz kommt zu dem Ergebnis, dass sich die Arbeitsstellen zu Organisationseinheiten entwickelt haben, in denen es vornehmlich um den Einbau von wissenschaftlicher, vor allem jedoch darauf basierender konzeptioneller Kapazität als wissenschaftlich informierter Politikformulierung in den zentralen Bildungsbehörden geht (Huberman, 1989). Vorrangige Absicht der politischen Entscheidungsinstitutionen war die Informationssammlung über den Systemzustand und - so bemerkenswert die schliesslichen Strukturveränderungen angesichts der politischen Widerstände waren - eher kleinteilige, unmittelbar wirksame Lösungen dringlicher Probleme (siehe oben). Weil zwischen Zustandsanalyse und Handlungskonsequenzen aber kein deduktiver Zusammenhang besteht, waren umfassendere Ansätze von Bildungsplanung schon aus «System»- und institutionellen Gründen nur schwer zu realisieren, weil politische Dezision wissenschaftlich gebotene Handlungskonsequenzen «brechen» kann. Planungsansätze, die auf alternative Ziel- und Massnahmenklärung und Generierung von Entscheidungssituationen abzielten, gerieten in Widerstreit zur politisch im Status quo beharrenden Mehrheit (und konnten im Lauf der Jahre nur in antizipatorische Vorwegnahme derartiger Bedenken umschlagen). Insofern veränderten sich auch die Funktionsprioritäten von «bildungswissenschaftlicher» Analyse und Beratung zur Vorlage von wissenschaftlich informierten und politisch begründeter «Konzeptionen» (PA ZH, 1972, S. 3) zur Plausibilisierung und Legitimierung der Schulpolitik.

Allerdings ist diese Einschätzung von «strengen» Definitionsmassstäben von Bildungsplanung abhängig. Denn zweifellos der Bildungsplanung zuzurechnen ist die anfängliche Konzentration der Arbeitsstellen auf die Strukturen der Sekundarstufe I als einem Ausschnitt des Schulsystems. Damit untrennbar verbunden waren Planungsaufgaben hinsichtlich der schulischen Kapazitäten (Aufnahmefähigkeit, Ausbaunotwendigkeiten, Bestandsfragen), der Curricula (Anforderungen an Schulformen, an Jahrgangsstufen, Lektionentafeln etc.), der Standorte (räumliche Verteilung der Schulen, «Betriebsgrössen» etc.) und schliesslich der Finanzen (Personal- und Sachmittel, Investitionen). 


\section{Handlungsfähigkeit und Selbstlegitimierung}

Aus den Aufgabenbeschreibungen wird deutlich, dass die Strukturthematik, nicht von vornherein als Arbeitsschwerpunkt feststand. Sie «ergab» sich als «willkommene» Gelegenheit in einem Prozess der binnenorganisatorischen Zuweisung von und Suche nach Aufgaben, Kompetenzen, faktischer Handlungsfähigkeit und Selbstlegitimierung (neben anderen Aufgaben, formal Bestandsaufnahmen, Dokumentation und Bildungsstatistik, inhaltlich Neufassung der Lehrpläne, Lehrerbildung, Analyse von Unterrichtsformen, Konzepte zur Gestaltung der interkulturellen Pädagogik etc.; vgl. auch Kohler 1973). Was aus einer gesellschaftlichen und vor allem schulischen Perspektive als «die» unitarische und hierarchische «Erziehungsdirektion» erscheint, war aus der Innenperspektive hochdifferenziert und wurde durch den organisatorischen "Anbau» der Arbeitsstellen zusätzlich pluralisiert. Es war weder mit ihrer Gründung getan noch mit einer Kompetenz- und Aufgabenzuweisung als "Amtsauftrag» und Handlungsressource. Die Arbeitsstellen mussten ihren Platz in den bestehenden Behörden finden und sich in diese «Verwaltungsökologie» einpassen, d.h. über den Amtsauftrag zugewiesene Legitimität musste durch Akte der Selbstlegitimierung ergänzt werden. Über die formale Ressourcenausstattung hinaus (Aufgabenbeschreibung, Zuständigkeiten, Personal etc.) waren die Arbeitsstellen dazu veranlasst, ihre reale Handlungskompetenz durch Bewährung in der Bearbeitung von Aufgaben zu beweisen, um intern als legitim akzeptiert zu werden und die eigene Bestandserhaltung zu gewährleisten. Das Strukturthema aufzugreifen, war deshalb eine strategische Option der neuen Arbeitsstellen, um sich eine - gegenüber anderen Themen und Organisationseinheiten - relativ exklusive Aufgabe zu sichern und darin ihre Handlungsfähigkeit auszudrücken. Dazu kam, dass in einer sachlichen Perspektive das Strukturthema dringlich war, vor allem in den Folgen expansiver Ausdehnungsprozesse, aber auch im Blick auf den Zusammenhang von Schulstrukturen und Prozessen der Steigerung des Qualifizierungsniveaus und der sozialen Statuszuweisung (Chancengleichheit).

Weil die Erziehungsdirektionen und ihre Abteilungen aber nicht auf die Arbeitsstellen "gewartet» hatten, resultierten aus ihrer Verflechtung mit der «alten» Organisation mit noch dazu gegenüber routinisiert, unmittelbar praxisorientiertem Verwaltungshandeln konkurrierenden Rationalitäts- und Relevanzkriterien Kompetenzprobleme und «Machtkämpfe». So war etwa das AfU in Bern nur für materielle Strukturfragen zuständig, während die Genehmigung der dann stattfindenden Schulversuche dem «alten» Amt für Unterricht - auf Antrag der Gemeinden - vorbehalten war. Und in Zürich kam es zwischen der PA und der Abteilung Volksschule zu einem «Gerangel» um die Zuständigkeit für die Lehrplanrevision, wobei letztere sich schliesslich durchsetzte.

In den strategischen «Lösungen» dieser Kompetenz- und Legitimationsfragen unterschieden sich die Kantone Bern und Zürich aber beträchtlich. Das AfU in Bern drängte nicht darauf, Entscheidungssituationen für kantonsweit generalisierte Politikkonzepte zu «erzwingen», sondern wartete auf bzw. hielt «Ausschau» 
nach Problemschulen für Strukturversuche, die mit den Ergebnissen bildungswissenschaftlicher Erkenntnisse verklammert werden konnten. Es stand deshalb weniger unter Handlungsdruck und konnte wissenschaftlicher agieren. Wissenschaftlich-administratives Handeln wartete auf "reife» Entscheidungssituationen, d.h. die herkömmlich gedachte Richtung, wonach Probleme politische Lösungskonzepte herausfordern (wie in Zürich) war «umgedreht»: Lösungskonzepte kooperativer und integrativer Schulmodelle, die das AfU erarbeitet hatte, "warteten" auf die "passenden" Probleme (Kingdon, 1995, S. 173 ff., S. 221 ff.). Das AfU legitimierte seine Arbeit und bewies Handlungsfähigkeit über sein Lösungsangebot für dringliche Bedürfnisse von Gemeinden, deren Sekundarstufenschulen unter Funktions- und Bestandsproblemen litten (Gemeindeprobleme als Handlungsressource). Durch die Arbeitsstelle wurde der Politikmodus keineswegs umfassend auf konzeptionelle, kantonsweite "Planung» umgestellt. Im Ergebnis konnte das AfU wissenschaftlich(er) als die PA in Zürich arbeiten ${ }^{7}$, freilich um den Preis, den kantonalen politischen Prozess der Gesamtrevision der Bildungsgesetzgebung, der parallel zu den Schulversuchen lief, kaum wesentlich beeinflussen zu können (Kussau, 1998).

Die PA in Zürich handelte "planhafter» im Blick auf den Entwurf eines Politikkonzepts zur Problemlösung und setzte ihre Handlungsfähigkeit und legitimatorische Akzeptanz durch ihre systematische Schulversuchsanlage einem erheblich höheren Erfolgsdruck aus. Sie war darauf angewiesen, Schulen für die Versuchsteilnahme als Erfolgsausweis zu suchen und zu finden. In dem Augenblick, in dem das "nach Plan» schwer fiel, geriet die PA in Legitimationsnöte. Ausserdem war diese Strategie auf die vorbehaltlose Unterstützung ihrer politischen Leitung (Erziehungsdirektor) angewiesen; da sie nicht durchgängig gegeben war, büsste sie an legitimierter Handlungsfähigkeit ein. Die Gemeinden in Zürich waren nur eine potentielle Handlungsressource, die aufgrund der hohen Schulgemeindeautonomie auch ausfallen konnte (und jahrelang ausfiel bzw. erst erschlossen werden musste). Die PA lief in der Strukturfrage in das Risiko eines «Ressourcenlochs» hinsichtlich Handlungskompetenz und Selbstlegitimierung und musste unter relativem Verzicht auf Wissenschaftlichkeit politisch-konzeptionelle Überzeugungsarbeit leisten.

\section{Bedeutung und Wirkung der Planung der Pädagogischen Arbeitsstellen}

Die Frage nach der Bedeutung von Bildungsplanung schliesst an den Zusammenhang zwischen gesellschaftpolitischer Funktion von und Erwartungen an Planung und ihrer binnenorganisatorischen Etablierung in den Erziehungsdirektionen an. In welchem Ausmass ist die Schulpolitik tatsächlich um neue informatorische Planungskomponenten ergänzt worden? 


\section{Begrenzte Informationen}

Seit den Anfängen von Bildungsplanung kommt Informationen eine Schlüsselrolle in der Politik zu. Die durch Verwissenschaftlichung erreichte Informationsvermehrung ändert indes nichts am notorischen Informationsdefizit der Politik. Auf wissenschaftlichen Informationen gegründete Planung unterliegt systematischen endogenen Restriktionen. Zu nennen sind begrenzte Kapazitäten der Informationssuche, -sammlung und -verarbeitung, Informationskosten und eine zirkuläre Logik der Informationssuche: je mehr Informationen verfügbar, desto grösser der Informationsbedarf bei gleichzeitig fehlenden, inhärenten Stoppregeln für Suchprozesse mit der Folge, Informationsansprüche auf ein brauchbares («satisficing»), jedoch "willkürliches» Mass herunterzuschrauben. Im Ergebnis muss die Politik weiter unter Bedingungen unvollständiger Informationen über verfügbare Handlungsalternativen unter Ungewissheit riskant entscheiden («bounded rationality»; Simon, 1972) - in der Strukturfrage auch durch «Informationsverweigerung» politischer Mehrheiten gespeist, um «kognitiver Dissonanz» mit dem an Statusbewahrung orientierten Selbstbild aus dem Wege zu gehen. Diese Feststellungen gelten umso mehr, wenn die Informationsfunktion eher sozialtechnologisch angelegt und nicht auf kommunikative Verständigung hin orientiert ist (Habermas, 1969).

\section{Exogene Restriktionen der Planung}

Aus der Planungs- und Informationsperspektive erscheint Politik - im Folgenden verstanden als entscheidungsbefugtes Institutionensystem (Erziehungsdirektor, Regierungsrat, Parlament, Volk) - als exogener Faktor. Politik wird zur «äusseren» Restriktion politischer Planung, die auf der Grundlage systematischer Informationen «weiss, was richtig ist». Demokratiepolitisch, aber auch im Sinn politischer Problemlösung, muss die Frage freilich andersherum gestellt werden: Sind unzureichende Informationen eine Restriktion rational-problemlösender Politik - volkssouveräner Politik, die auch gegen ihre «wohlverstandenen» Interessen abstimmen kann - und kann Politik informatorisch rationalisiert werden? Die Antworten auf diese Frage fallen zwiespältig aus.

\section{«Alte» und neue Steuerungsinformationen}

Einen ersten Zugang zur Beantwortung der Frage gibt die Geschichte der Strukturreformen. Aus dem vermeintlichen Gegensatz zwischen ökonomischen Qualifikationsinteressen und Demokratisierungsideen erwuchs die stärkste Schubkraft für die Strukturreformen. Denn Ausschöpfung eines Begabungsreservoirs setzte Demokratisierung mindestens der schulischen Zugänge ebenso voraus wie umgekehrt Qualifizierungsinteressen in einer Ausweitung der Bildungsteilhabe an weiterführenden Bildungsabschlüssen münden mussten. Diese historisch unwahrscheinliche Gemeinsamkeit «erzwang», wenn auch erst nach langen politischen Auseinandersetzungen, doch die Strukturreformen gegen einen politischen und kulturellen Konservatismus, der zunächst gegenüber jeglicher 
geplanter, demokratisierender Initiative Oberhand gewonnen hatte, als die Wachstumsphase in eine Rezession umschlug (König, 1998).

Diese Mehrheit geriet politisch in die Minderheit, kaum durch wissenschaftliche und argumentative Aufklärung, d.h. Informationen und Argumente setzten sich nicht umstandslos durch, zumal sie in ihrer Bedeutung immer mit gleichzeitigen sozioökonomischen, schulpolitischen und schulischen Veränderungen, Wandel normativer Orientierungen und politischer Diskurse abgeglichen werden müssen. Wissenschaftliche Informationen mussten vielmehr mit «alten» Handlungsorientierungen konkurrieren, die, wie unmodern sie auch sein mochten, Wirksamkeit behielten. Informationsbasierte Planung bekam im Zuge der Rationalisierung und Verwissenschaftlichung der Gesellschaft zwar grössere Bedeutung, optimistisch gedacht als Substitution «rückständiger» Handlungsmodelle. Jedoch stand (und steht) sie - wie im nachhinein deutlicher sichtbar weiterhin in Konkurrenz zu politisch relevantem Orientierungswissen wie kulturellen Traditionen, Alltagswissen, eingeschliffenem Institutionen- und Regelwissen und schliesslich auch ideologischen und interessenbezogenen Positionen (Lindblom \& Cohen, 1979, S. 10 ff.). Wissenschaft muss sich unter (direkt)demokratischen Bedingungen mit ihrer spezifischen Rationalität in die jeweiligen institutionellen Prozessformen mit ihren unterschiedlichen, entscheidungsrelevanten Informationsquellen einfügen oder versuchen, den öffentlichen Diskurs zu beeinflussen, dann jedoch unter Umständen mit langen Latenzzeiten rechnen.

Trotzdem lassen sich Effekte der informatorischen Verwissenschaftlichung ausmachen. Informationen wirken nicht direkt und unmittelbar, ausser unter bestimmten Bedingungen, wenn sie sich z.B. in den öffentlichen und politischen Mehrheitsdiskurs einfügen. Umgekehrt sind Informationen selbst durch normative Bezüge in den politischen Prozess eingebunden. Informationsorientierte Planung muss politische Durchsetzungsmassstäbe berücksichtigen. Zwischen Politik und Information haben jedoch «Sickerprozesse» stattgefunden. Es ist im Prozess der «Verwissenschaftlichung» der Politik "verwehrt», uninformiert und ohne rational-wissenschaftliche Begründung zu agieren - mindestens als Nutzung des «Prestige(s) der Wissenschaften» (König et al., 1998, S. 19). «Policy-Lernen» ist indes nur möglich, wenn Politik und - in direktdemokratischen Systemen - die Bürgerinnen und Bürger lernen, mit Informationen und argumentativen Begründungen in öffentlichen Diskursprozessen umzugehen. Allerdings kann niemand auf diese Lernaufgabe verpflichtet werden, vielmehr ist "strategische», systematisch in die Gesellschaftsformation eingebaute Selektivität zu erwarten (Offe, 1972b). Informationen unterliegen dem Risiko, politisch-anwendungsorientiert zu Legitimationszwecken vereinnahmt (Huberman, 1989, S. 81) oder auch schlicht negiert zu werden. Denn Informationen sind nicht einfach «gut» und «hilfreich», sie besitzen «dual use»-Eigenschaften. Ihre Qualität und Problemlösungsrelevanz erhalten sie erst in spezifischen selektiven Deutungskontexten. «Expertenwissen ist grundsätzlich Wissen über Umwelten» (Baecker, 1999, S. 75, S. 74 ff.). Nur als solches werden Informationen wahrge- 
nommen, weil sie der Politik Verfügungsrechte und die Entscheidungsoption darüber lassen. Gerade aus der Informationsperspektive erscheinen politische Entscheide immer als Dezision und Ausdruck einer Rationalitätskrise - mit zwei möglichen Konsequenzen. (1) Wird Information zur Entscheidungsgrundlage, wechselt sie die Systemreferenz; sie wird selbst zur «Politik». (2) Bleibt Wissenschaft aber Informationen produzierende Wissenschaft und planen Planungsabteilungen, dann behält die Politik sich den Verwertungsprimat vor. Sie besteht auf Vorlage von «alternativen Entscheidungsgrundlagen» und verweigert sich wissenschaftlicher «Sachgesetzlichkeit». Die Informationsfunktion wird also systematisch enttäuscht, erst recht in direktdemokratischen Systemen. In diesem Sinne war auch der Anbau der Organisationsform «Pädagogische Arbeitsstellen» nicht Ausdruck eines Wandels im Politikmodus hin zu Planung, sondern sozialtechnologischer Modernität - mit selektiven Verwendungsoptionen. Die Arbeitsstellen fanden sich genau in dieser Ambivalenz der Verwendung von Informationen. Sie waren als "wissenschaftliche» Problemlöser gedacht, mit dem Risiko der Irrelevanz, mussten aber gleichzeitig den Standpunkt «politischer» Problemlöser - ohne Entscheidungskompetenz - einnehmen (Lindblom \& Cohen, 1999, 11), mit dem Risiko, in politischen Auseinandersetzungen verschlissen zu werden.

Die Diskussion um Information und Planung kreist um die Pole: Information zur Problemlösung (Lindblom \& Cohen, 1979; SKBF, 1996, S. 47) und Information zur Legitimierung politischer Entscheide. Wenn man unterschiedliche Rationalitäts- und Relevanzmassstäbe zwischen «Politik» und (wissenschaftlicher) «Information» anerkennt, spricht viel dafür, dass Informationen ebenso sehr Legitimierungs- wie Problemlösungsfunktionen haben, wenn sie sich selbst als spezifische Beiträge in politischen Prozessen "politisieren». Mindestens die Erfahrungen der Arbeitsstellen weisen in diese Richtung, wenn sie sich konzeptionell-konstruktiv ausrichten.

\section{Informationsbasierte Planung und ihre direktdemokratische Grenzen}

Wenn es heisst, «dass Bildungsplanung kein geeignetes Instrument zur Lösung gesellschaftspolitischer Konflikte darstellt, sondern dass die [politische; die Verfasser] Polarisierung ihr die Grundlagen entzieht» (v. Friedeburg \& Oehler, 1984, S. 257 f.), so ist das ebenso richtig wie verkürzt, weil Planung in der Strukturfrage selbst in Politik «aufging». Planung entwickelte keinen eigenständigen Politikstatus, sondern war hin- und hergerissen zwischen informationsbasierter, empirischer Sachlichkeit («Richtigkeit») und Berücksichtigung politisch-gesellschaftlicher Bedingungen, und die Arbeitsstellen verstanden sich als "politische» Reformer. Die «Entdeckung» der politischen Handlungsressource Information für Planung und deren Funktionalisierung wurde selbst zum politischen Streitthema. Glaubten die einen an die Herrschaft der «Sachgesetzlichkeit» (Schelsky, 1979, bes. S. 464 ff.; kritisch Habermas, 1969) und damit an eine technokratische Entpolitisierung gesellschaftlicher und politischer Entwicklung ${ }^{8}$, so setz- 
ten die anderen auf demokratisierende Aufklärung und Teilhabe an politischen Prozessen sowohl auf Seiten des Staates durch «Interessenberücksichtigung" (Mayntz \& Scharpf, 1973, S. 141 ff.) als auch auf Seiten der betroffenen und beteiligten Bürgerinnen und Bürger («Neue soziale Bewegungen») bzw. im Schulkontext der Kinder und Eltern (Deutscher Bildungsrat, 1973). Denn es zeichnete sich mehr und mehr ab, dass der Staat zur Durchsetzung und Legitimierung seiner Politik auf die Kooperation nicht-staatlicher Akteure angewiesen war und spezifisch in der Schulpolitik auf die zwar «staatlichen» Akteure der Lehrerinnen und Lehrer, die aber nicht (ausschliesslich) als «politisches» Personal definiert waren.

Nicht zuletzt institutionelle Grundbedingungen der Referendumsdemokratie (Möckli, 1994; Neidhart, 1970) liessen - wie gerade die politisch konfliktiven und langwierigen Strukturreformen zeigen - «Sachgesetzlichkeit» als Darstellung kausaler empirischer Zusammenhänge gar nicht erst politisch bedeutsam werden. Vielmehr wurden selbst «beste» Argumente und Untersuchungen entweder mit anderen «besten» Argumenten oder Untersuchungen gekontert - empirische Information eingefügt in normative politische Deutungskontexte wird selbst zur "Meinung». «Politik schlug» Information allemal, denn ein Kennzeichen von Schulpolitik ist, dass sie "Politik» ist. "Die Diskussion um die Gesamtschule schläft ein, in der Öffentlichkeit ist sie diskreditiert, sie wird als politische Frage angesehen. Das müsste nicht sein» (Interkantonale Studiengruppe «Gesamtschule», 1975, zitiert nach Wymann, 1993, S. 156). Gleichzeitig wurde Information in Politik (-vorbereitung) integriert und entsprechend funktionalisiert. Besonders das Referendumssystem wendet das Verhältnis zwischen Politik und Information eher zu Lasten der Information. Direktdemokratien sind institutionell nicht planungsfreundlich, weil jede Planung grundsätzlich unter dem Vorbehalt nachträglicher Volksabstimmungen, in denen sämtliche Sorten von Informationen samt spezifischen Bewertungskriterien eine Rolle spielen, steht, die vorgängige Planungsaufträge und -leistungen in ihrer Verlässlichkeit entwerten kann. Denn das direktdemokratische System kennt, anders als konkurrenzdemokratische Systeme, bis zur jeweiligen Schlussabstimmung keine zuverlässig vorhersehbaren Mehrheiten; selbst vorparlamentarische Absprachen und Kompromisse können in Volksabstimmungen verworfen werden - das «Volk als Störfaktor in der Planung?» (Hotz \& Werder, 1979). In diesem System haben Planungsleistungen nur dann Aussicht auf Realisierung, wenn sie Anschluss an den mehrheitsfähigen Diskurs finden (bei gleichzeitig begrenzten Möglichkeiten, darauf Einfluss zu nehmen).

\section{Ausblick: Dezentralisierung und Auslagerung}

Seit der Gründung der Arbeitsstellen in Bern und in Zürich sind beinahe dreissig Jahre vergangen. Über ihre gegenwärtige organisatorische Stellung und funk- 
tionale Bedeutung kann hier nichts gesagt werden. Die funktionale Relevanz von Informationen für Politik ist hingegen unumstritten. Die Politik kann hinter das erreichte Niveau der Informationsverarbeitung nicht mehr zurück. In diesem Sinne hat die Institutionalisierung der Informations- und Planungsfunktion eine sachlich fundierte Politikformulierung gefördert. In den letzten Jahren zeichnet sich eine neue Entwicklung entlang zweier Dimensionen ab, die auf den Achsen «zentral - dezentral» (1) und «intern - extern» (2) liegen. Dadurch könnte sich das Verhältnis zwischen Politik und Information neu einstellen (wobei die Erfahrungen der Praxis abzuwarten bleiben).

(1) Die Schulentwicklungs- und Autonomiepolitik hat die Diskussion um zentrale Interventionen der kantonalen politischen Institutionen belebt und auf stärkere Dezentralisierung umgestellt. Zunächst freilich nur programmatisch wird zentralistischen Reformstrategien, wie noch bei den Strukturreformen verfolgt, eine Absage erteilt (Oertel, 1993). Gleichzeitig sind aber, begünstigt durch das am New Public Management angelehnte Konzept der «wirkungsorientierten Verwaltungsführung» zwei eher gegenläufige Entwicklungen erkennbar: Zum einen ist der kantonale (zentrale) Aufbau von Qualitätssicherungssystemen (Reorganisation und Funktionsumstellung der Schulaufsicht) vorgesehen. Zum anderen weist die «Wiederentdeckung der Schule als pädagogische Gestaltungsebene» (Fend, 1988) ein zentralistisches Komplement auf, weil kantonale Vorgaben zur Steuerung der (dezentralen) Einheit Schule vorgesehen sind (was sich auch in der zentralen Einführung von Schulautonomie ausdrückt). Es zeichnet sich ab, dass zentrale, "strategische» Schulpolitik längst nicht ausgedient hat und die Dezentralisierungsabsichten einer empirischen Bestätigung harren.

(2) Besonders gefördert durch die Ausrichtung der Politik am New Public Management-Konzept wird die Informationsfunktion - «Beratung» und «Evaluation» - in der Tendenz extern ausgelagert. Ein Problem, das mit dem Einbau von Sachverstand in die Arbeitsstellen gerade "gelöst» werden sollte, kehrt jedoch mit dem "outsourcing» zurück, nämlich die Zusammenarbeit mit Externen, die zwar kompetent sein mögen wie die internen Fachleute, aber «zu kritisch, unzuverlässig, arrogant, wortreich oder [...] gleich alles zusammen» (Huberman, 1989, S. 69). Auch hier wird man zusehen müssen, ob das Beratungs- und Evaluationsgeschäft in der Interaktion mit dem politisch-administrativen System neue Vermittlungsformen zwischen "Praxis» und "Wissenschaft» hervorbringt und welche Leistungsfähigkeit ein derartiges Informationssystem entwickeln kann.

Kaum vermeidbar werden freilich wechselseitige Enttäuschungen sein, weil das politische Entscheidungssystem sich die Verfügungsrechte über den Umgang mit Informationen vorbehalten muss. Insofern können die mit dem Einbau informationsgestützter Planung (in Form der Arbeitsstellen) verknüpften Erwartungen an die wissenschaftliche Leistungsfähigkeit für die Politik mindestens in der Strukturfrage als «Missverständnis» interpretiert werden. Die Politik erwartete «neutrale» wissenschaftliche Informationen und bekam wissenschaftlich be- 
gründete Reformnotwendigkeiten (vor einem spezifischen normativen Hintergrund) - mit der Folge partieller Verweigerung von Informationen. Wissenschaft ist in ihrem "Eigensinn» (a) für die Politik nicht berechenbar und sogar latent «subversiv» (ausser sie "dient» sich der Politik an) und (b) müssen wissenschaftliche Informationen politisch übersetzt und praktisch handhabbar gemacht werden, da Wissenschaft nicht unmittelbar politische Entscheide begründet; erst dann kann die Leistungsfähigkeit der Wissenschaft ausgeschöpft werden. Diesem Risiko eines Missverständnisses unterliegt neuerdings vermehrt ausgelagerte Wissenschaft in gleicher Weise. Denn weniger bedeutsam als die Dimension «intern-extern» ist das Verhältnis zwischen Politik und Wissenschaft in ihren wechselseitigen Erwartungen: Was erwarten Politik und Pädagogik (schulische Praxis) von erziehungs- und sozialwissenschaftlicher Forschung und was kann umgekehrt die Wissenschaft anbieten? Ein als «Lernsystem», auf Evaluation gegründetes, (wissenschaftliche) Rationalität verbürgendes politisches System ist formal organisierbar. Es setzt allerdings Lernbereitschaft voraus und ist - einschliesslich der Wissenschaft selbst - weiterhin eingebunden in "Politik», in unterschiedliche Situationsdeutungen, Problemdefinitionen, Handlungsorientierungen, Interessenlagen, Ideologien und Wertbezüge.

\section{Anmerkungen}

1 Alte Bundesverfassung, Art. 27, Abs. 2. Die einschränkende Formulierung «Primarunterricht» wurde damals noch mehrheitlich dahingehend interpretiert, dass sie auch die Sekundarstufe I mit einschloss.

2 Lutz (1983, S. 222) stellt die Frage, ob die Entwicklung der Dienstleistungsgesellschaft das Ergebnis eines kontinuierlichen Modernisierungsprozesses ist oder einen Strukturbruch darstellt.

3 Seither fortgeschrieben als postindustrielle Gesellschaft und heute als Informations- und Wissensgesellschaft. Allen Beschreibungen gemeinsam ist ihre schulpolitische Wahrnehmung als steigende Qualifikationsanforderungen.

4 Nicht untypisch ist die Aussage von Fritz Hummler (Bundesdelegierter für Arbeitsbeschaffung) im Jahr 1963 zur Frage der Ausschöpfung der Begabungsreserven, die sich in dem Satz zusammenfassen lässt, niemand solle auf einmal zu hoch hinaus wollen (Zitat bei Horvath, 1998, S. 86).

5 Diese kantonal-zentralistische Perspektive beschreibt vor allem den Zürcher Planungsansatz. Die Berner Schulversuche zeigen in ihrem Bezug auf örtliche Schulprobleme Merkmale einer lokalen «Schulstrukturentwicklung».

6 Wenn heute eher vom «aktivierenden» Staat die Rede ist, kommt in dieser semantischen Formel das veränderte Funktions- und Steuerungsverständnis des Staates zum Ausdruck - weg von hierarchisch-unilateraler «Verordnung» hin zu heterarchischen Interventionsformen.

7 Die Unterschiede zeigen sich auch in der Publikationspraxis der beiden Arbeitsstellen. Während der Kanton Bern seine Unterlagen auch einer kantonsübergreifenden Öffentlichkeit zur Verfügung stellen kann, scheidet diese Variante in Zürich weitgehend aus, weil es sich um einer breiteren Öffentlichkeit nicht zugängliche Politikentwürfe handelt.

8 «[...] less and less political decisions, more and more administrative ones» (Moynihan, 1965/66, S. 12). 


\section{Literatur}

Allmendinger, J. (1989). Educational Systems and Labor Market Outcomes. European Sociological Review, 5, 231-250.

Baecker, D. (1999). Zum Problem des Wissens in Organisationen. In D. Baecker, Organisation als System. Aufsätze (S. 68-101). Frankfurt a.M.: Suhrkamp.

Baethge, M. \& Teichler, U. (1984). Bildungssystem und Beschäftigungssystem. In: M. Baethge \& K. Nevermann (Hrsg.), Enzyklopädie Erziehungswissenschaft Bd. 5: Organisation, Recht und Ökonomie des Bildungswesens (S. 206-225). Stuttgart: Klett-Cotta.

Baumert, J. (1991). Langfristige Auswirkungen der Bildungsexpansion. Unterrichtswissenschaft, 19, 333-349.

Bern-Dekret (1971). Gemeinsamer Antrag des Regierungsrates und der Kommission vom 23. Juni/14. Juli und 23. Juni 1971: Dekret über die Organisation der Erziehungsdirektion. Bern: s.n.

Bernischer Lehrerverein (Hrsg.). (1972). Gesamtschule. Praktische Aspekte der inneren Schulreform. Bern \& Stuttgart: Haupt.

Bornschier, V. \& Aebi, D. (1992). Rolle und Expansion der Bildung in der modernen Gesellschaft. Von der Pflichtschule bis zur Weiterbildung. Schweizerische Zeitschrift für Soziologie, 18, (3), 539-567.

Buchmann, M. \& Sacchi, St. (1998). The Transition from School to Work in Switzerland. Do Characteristics of the Educational System and Class Barriers Matter? In Y. Shavit \& W. Müller, with the editorial assistance of C. Tame (eds.), From School to Work. A Comparative Study of Educational Qualifications and Occupational Destinations (S. 407-442). Oxford: Clarendon Press.

Criblez, L., Hofstetter, R., Jenzer, C. \& Magnin, Ch. (Hrsg.). (1999). Eine Schule für die Demokratie. Zur Entwicklung der Volksschule in der Schweiz im 19. Jahrhundert. Bern, Berlin, Bruxelles, Frankfurt a.M., New York \& Wien: Lang.

Cohen, S. S. (1970). From Causation to Decision: Planning as Politics. The American Economic Review (Papers and Proceedings), 60, 180-185.

Czada, R. \& Schimank, U. (2000). Institutionendynamiken und politische Institutionengestaltung: Die zwei Gesichter sozialer Ordnungsbildung. In R. Werle \& U. Schimank (Hrsg.), Gesellschaftliche Komplexität und kollektive Handlungsfähigkeit (S. 23-43). Frankfurt a.M. \& New York: Campus.

Dahrendorf, R. (1968 [1965]). Bildung ist Bürgerrecht. Plädoyer für eine aktive Bildungspolitik. Hamburg: Christian Wegner.

Deutscher Bildungsrat (1974). Bericht der Bildungskommission zur Reform von Organisation und Verwaltung: Fragen einer ziel- und programmorientierten Schulverwaltung unter besonderer Berücksichtigung des Ministerialbereichs. Verabschiedet auf der 38. Sitzung der Bildungskommission am 13./14. Februar 1974 in Bonn. Bonn: Deutscher Bildungsrat.

Ekholm, M. (1997). Steuerungsmodelle für Schulen in Europa. Schwedische Erfahrungen mit alternativen Ordnungsmodellen. Zeitschrift für Pädagogik, 43, 597-608.

Erziehungsdirektion des Kantons Zürich (1990). Entwicklung der Oberstufe der Volksschule und des Gymnasiums. Eine Statistische Bestandesaufnahme und Lageanalyse. Bericht der internen Arbeitsgruppe. Zürich: Erziehungsdirektion.

Fend, H. (1988). Schulqualität - die Wiederentdeckung der Schule als pädagogische Gestaltungsebene. Neue Sammlung, 28, 537-547.

Friedeburg, L. von \& Oehler, Ch. (1984). Staatliche Bildungsplanung. In M. Baethge \& K. Nevermann (Hrsg.), Enzyklopädie Erziehungswissenschaft. Bd. 5: Organisation, Recht und Ökonomie des Bildungswesens (S. 244-258) Stuttgart: Klett-Cotta.

Fuchs, P. (1999). Intervention und Erfahrung. Frankfurt a.M.: Suhrkamp.

Goodin, R. E. (1996). Institutions and Their Design. In R. E. Goodin (ed.), The Theory of Institutional Design (S. 1-53). Cambridge, New York \& Melbourne: Cambridge University Press. 
Graf, M. \& Lamprecht, M. (1991). Der Beitrag des Bildungssystems zur Konstruktion von sozialer Ungleichheit. In V. Bornschier (Hrsg.), Das Ende der sozialen Schichtung? Zürcher Arbeiten zur gesellschaftlichen Konstruktion von sozialer Lage und Bewustsein in der westlichen Zentrumsgesellschaft (S. 73-96). Zürich: Seismo.

Habermas, J. (1969). Verwissenschaftlichte Politik und öffentliche Meinung. In J. Habermas, Technik und Wissenschaft als "Ideologie» (S. 120-145). Frankfurt a.M.: Suhrkamp.

Hedinger, U. K. \& Wyttenbach, S. (1984). Zur Entwicklung der Schulstrukturen: Strukturreformen und Schulversuche in der Schweiz. Bern: EDK (=Informationsbulletin 45a).

Horvath, F. (1998). Im Windschatten der Wissenschaftspolitik. Ständisch-föderalistische Interessenentfaltung im Zeichen des «Bildungsnotstands». In M. König, G. Kreis, F. Meister \& G. Romano (Hrsg.), Dynamisierung und Umbau. Die Schweiz in den 60er und 70er Jahren (S. 81-93). Zürich: Chronos.

Hotz, B. \& Werder, H. (1979). Planung im politischen Entscheidungsprozess. Hauptprobleme und Befunde. In W. Linder, B. Hotz \& H. Werder, Planung in der schweizerischen Demokratie. Ein Forschungsprojekt des Instituts für Orts-, Regional- und Landesplanung der ETH Zürich (S. 81-114). Bern \& Stuttgart: Haupt.

Huberman, M. (1989). «Situationsanalyse» der Pädagogischen Abteilungen der Kantone in der Deutschschweiz. Bildungsforschung und Bildungspraxis, 11, 66-82.

Jenzer, C. (1997). Schulstrukturen als historisch gewachsenes Produkt bildungspolitischer Vorstellungen. Blitzlichter in die Entstehung der schweizerischen Schulstrukturen. Bern, Berlin, Frankfurt a.M., New York, Paris \& Wien: Peter Lang.

Kingdon, J. W. (1995). Agendas, Alternatives, and Public Policies. (2. Aufl.). New York: Harper Collins College Publishers.

König, M. (1998). Politik und Gesellschaft im 20. Jahrhundert. Krisen, Konflikte, Reformen. In M. Hettling, M. König, M. Schaffner, A. Suter \& J. Tanner, Eine kleine Geschichte der Schweiz. Der Bundesstaat und seine Traditionen (S. 21-90) Frankfurt a.M.: Suhrkamp.

König, M., Kreis, G., Meister, F. \& Romano, G. (1998). Einleitung: Reformprojekte, soziale Bewegungen und neue Öffentlichkeit. In M. König, G. Kreis, F. Meister \& G. Romano (Hrsg.), Dynamisierung und Umbau. Die Schweiz in den 60er und 70er Jahren (S. 11-20). Zürich: Chronos.

Kohler, S. (1973). Kurz- und mittelfristige Aufgaben der Erziehungsdirektion im bernischen Schulwesen. Amtliches Schulblatt, (Separatdruck), 1-12.

Kussau, J. (1998). Kanton Bern. Reform der Sekundarstufe I. NFP 33: Möglichkeit und Grenzen schulischer Reformentscheidungen am Beispiel der Reformbemühungen auf der Sekundarstufe I. Zürich [Manuskript].

Kussau J., Oertel L. \& Wohlgemuth A. (1998). Reform und Immobilismus im Schulwesen. Eine Politikstudie über Strukturreformen. NFP 33: Möglichkeit und Grenzen schulischer Re-formentscheidungen am Beispiel der Reformbemühungen auf der Sekundarstufe I. Zürich [Manuskript].

Lamprecht, M. \& Stamm, H. unter Mitarbeit von U. Meier (1996). Soziale Ungleichheit im Bildungswesen. Hrsg. Bundesamt für Statistik. Bern: Bundesamt für Statistik.

Leschinsky, A. \& Mayer, K. U. (eds.). (1990). The Comprehensive School Experiment Revisited: Evidence from Western Europe. Frankfurt a.M., Bern, New York \& Paris: Peter Lang.

Lindblom, Ch. E. \& Cohen, D. K. (1979). Usable Knowledge. Social Science and Social Problem Solving. New Haven \& London: Yale University Press.

Linder, W., Hotz, B. \& Werder, H. (1979). Planung in der schweizerischen Demokratie. Ein Forschungsprojekt des Instituts für Orts-, Regional- und Landesplanung der ETH Zürich. Bern \& Stuttgart: Haupt.

Lüscher, L. (1997). Geschichte der Schulreform in der Stadt Bern von 1968 bis 1988. Eine Analyse des Vorgehens und der Widerstände. Bern, Berlin, Frankfurt a.M., New York, Paris \& Wien: Peter Lang. 
Luhmann, N. (1993). Die Paradoxie des Entscheidens. Verwaltungs-Archiv, 84, 287-310.

Lutz, B. (1979). Die Interdependenz von Bildung und Beschäftigung und das Problem der Erklärung der Bildungsexpansion. In J. Matthes (Hrsg. im Auftrage der deutschen Gesellschaft für Soziologie), Sozialer Wandel in Westeuropa. Verhandlungen des 19. Deutschen Soziologentages, 17. - 20. April 1979 im Internationalen Congress Centrum (ICC) in Berlin (S. 634-670). Frankfurt a.M. \& New York: Campus.

Lutz, B. (1983). Bildungsexpansion und soziale Ungleichheit. Eine historisch-soziologische Skizze. In R. Kreckel (Hrsg.), Soziale Ungleichheiten. Soziale Welt, Sonderband 2 (S. 221245). Göttingen: Otto Schwarz.

Mayntz, R. \& Nedelmann, B. (1987). Eigendynamische soziale Prozesse. Anmerkungen zu einem analytischen Paradigma. Kölner Zeitschrift für Soziologie und Sozialpsychologie, 39, 648-668.

Mayntz, R. \& Scharpf, F. W. ( 1973). Kriterien, Voraussetzungen und Einschränkungen aktiver Politik. In R. Mayntz \& F. W. Scharpf (Hrsg.), Planungsorganisation. Die Diskussion um die Reform von Regierung und Verwaltung des Bundes (S. 115-145) München: Piper.

Möckli, S. (1994). Direkte Demokratie. Ein Vergleich der Einrichtungen und Verfahren in der Schweiz und Kalifornien, unter Berücksichtigung von Frankreich, Italien, Dänemark, Irland, Österreich, Liechtenstein und Australien. Bern, Stuttgart \& Wien: Haupt.

Moynihan, D.P. (1965/66). The Professionalization of Reform. The Public Interest, (1), 6-16.

Naschold, F. (1974). Schulreform als Gesellschaftskonflikt. Frankfurt a.M.: Athenäum.

Neidhart, L. (1970). Plebiszit und pluralitäre Demokratie. Eine Analyse der Funktion des schweizerischen Gesetzesreferendums. Bern: Francke.

Nordlinger, E. A. (1981). On the Autonomy of the Democratic State. Cambridge, Mass.: Harvard University Press.

OECD \& EDK (1989). Länderexamen Bildungspolitik. Schweiz I. o.O. [Paris \& Bern]: EDK.

Oertel, L. (1993) . Reform der Reformstrategie. Zur Umorientierung schulischer Innovationen. Bildungsforschung und Bildungspraxis, 15, 155-177.

Oertel, L. unter Mitarbeit von Ch. Aeberli, J. Handloser \& S. Rosenberg (1997). Schulreform - ein Zürcher Politikversuch. Zur Entwicklung der Volksschuloberstufe. Zürich: Orell Füssli.

Offe, C. (1972a). Strukturprobleme des kapitalistischen Staates. Aufä̈tze zur politischen Soziologie. Frankfurt a.M.: Suhrkamp.

Offe, C. (1972b). Klassenherrschaft und politisches System. Zur Selektivität politischer Institutionen. In C. Offe, Strukturprobleme des kapitalistischen Staates. Aufä̈tze zur Politischen Soziologie. (S. 65-105). Frankfurt a.M.: Suhrkamp.

PA ZH (1972). Pädagogische Abteilung der Erziehungsdirektion des Kantons Zürich. Rahmenplan für die Arbeit der Pädagogischen Abteilung. Zürich: Erziehungsdirektion.

PA ZH (1974). Pädagogische Abteilung der Erziehungsdirektion des Kantons Zürich: Die Pädagogische Abteilung der Erziehungsdirektion des Kantons Zürich. Schulblatt des Kantons Zürich, 89, 523-535.

Picht, G. (1965 [1964]). Die deutsche Bildungskatastrophe. München: DTV.

Rogger, F. (1992). Vom Zweiten Weltkrieg zur Gegenwart. In Bernischer Lehrerinnen- und Lehrerverein BLV: Jubiläumsband 100 Jahre BLV. Lehrerinnen und Lehrer zwischen Schule, Stand und Staat. Die Geschichte des Bernischen Lehrerinnen- und Lehrervereins (BLV) erarbeitet von P. Scandola, F. Rogger \& J. Gerber (S. 340-655). Bern: Bernischer Lehrerinnenund Lehrerverein BLV.

Rolff, H.-G. (1970). Bildungsplanung als rollende Reform. Eine soziologische Analyse der Zwecke, Mittel und Durchführungsformen einer reformbezogenen Planung im Bildungswesen. Frankfurt a.M., Berlin \& München: Moritz Diesterweg.

Schelsky, H. (1957). Schule und Erziehung in der industriellen Gesellschaft. Würzburg: Werkbund. 
Schelsky, H. (1979 [1961]). Der Mensch in der wissenschaftlichen Zivilisation. In H. Schelsky, Auf der Suche nach Wirklichkeit. Gesammelte Aufsätze zur Soziologie der Bundesrepublik (S. 449-499). München: Wilhelm Goldmann.

Schneider, R. (1982). Die Bildungsentwicklung in den westeuropäischen Staaten 1870-1975. Zeitschrift für Soziologie, 11, 207-226.

Simon, H. A. (1972). Theories of Bounded Rationality. In C. B. McGuire \& R. Radner (eds.), Decision and Organization. A Volume in Honor of Jacob Marschak (S. 161-176). Amsterdam \& London: North-Holland Publishing Company.

SKBF [Schweizerische Koordinationsstelle für Bildungsforschung] (1996). Was ist Bildungsforschung? Geschichte, Selbstverständnis und Perspektiven. Aarau: Schweizerische Koordinationsstelle für Bildungsforschung.

Stricker, H. (1991). Bildungskonzepte im Umbruch aus der Sicht der Bildungsforschung. Münchenwiler-Tagung vom 21./22. Juni 1991. Bern [Manuskript].

Swaan, A. de (1993). Der sorgende Staat. Wohlfahrt, Gesundheit und Bildung in Europa und den USA der Neuzeit. Frankfurt a.M. \& New York: Campus.

Weltbank (1997). Der Staat in einer sich ändernden Welt. Weltentwicklungsbericht 1997. Washington, D.C.: The World Bank.

Wolf, Ch., Jr. (1993). Markets or Governments. Choosing between Imperfect Alternatives. A RAND Research Study (2. Aufl.). Cambridge \& London: The MIT Press.

Wymann, H. (1993). Ausblick auf eine schulpolitische Wende. Die Oberstufe der Volksschule des Kantons Zürich von 1978-1992. Zürich: Werd Verlag.

\section{L'Expansion du secteur éducatif, réforme du niveau secondaire I et services de recherche pédagogique}

\section{Résumé}

Cette contribution traite des fonctions et des effets des services de recherche pédagogique dans les cantons de Berne et Zurich. Ceux-ci ont été institués dans les années soixante-dix dans le cadre des départements d'instruction publique. Ils devaient incorporer des informations scientifiques à la planification centrale comme nouveau mode politique. Du point de vue pratique ils devaient faire leurs preuves à propos des réformes du niveau secondaire premier degré, ce qui était une conséquence de l'expansion du secteur éducatif. Les fondements scientifiques de la réforme administrative étaient considérés comme une condition nécessaire de la réforme scolaire. L'information scientifique entrait rapidement en conflit avec les divergences politiques entre ceux qui voulaient l'ouverture fonctionnelle du système scolaire (démocratisation, qualification) et ceux qui défendaient le statu quo d'une éducation restrictive. Ce fait a hypothéqué la planification scolaire qui ne disposait d'une liberté d'action que dans la mesure où elle se soumettait aux raisons politiques. Si on constate une ouverture de la politique à la science, les informations scientifiques restent cependant tributaires des différents systèmes de valeur et options pratiques de la politique. 


\section{L'espansione della formazione, riforma del secondario I e servizi di ricerca pedagogica}

\section{Riassunto}

L'articolo si occupa delle funzioni e degli effetti dell'introduzione, avvenuta negli anni settanta, dei servizi di ricerca pedagogici istituiti all'interno dei dipartimenti dell'educazione nei cantoni di Berna e Zurigo. Il loro compito sarebbe stato quello di sostanzare la pia-nificazione centrale con informazioni a carattere scientifico, contribuendo così alla creazione di un nuovo metodo di azione politica. Inoltre avrebbero poi dovuto dimostrare la propria validità tra l'altro nell'ambito della riforma del secondario I, uno degli effetti dell'espansione della formazione. La riforma dell'amministrazione basata su conoscenze scientifiche venne infatti sì considerata quale premessa per la riforma scolastica, ma scivolò ben presto in una contraddizione politica tra l'apertura del sistema (democratizzazione e qualifiche) da un lato e la limitazione della formazione per fini conservatori dall'altro lato. Appesantita da questo fardello, la pianificazione della formazione poté assicurare la sua efficacia unicamente nella misura in cui seppe sottomettersi ai criteri della razionalità politica. Malgrado si possa osservare nella politica un aumento nella fruizione di conoscenze scientifiche, queste ultime restano sempre condizionate dai rapporti politici legati a valori e orientamenti diversi.

\section{Educational expansion, restructuring of the lower secondary school level and educational planning offices}

\section{Summary}

The paper presented refers to the functions and effects of educational offices in the public administration (ministry of education) of the Swiss cantons of Bern and Zürich that were established at the beginning of the seventies. These offices intended to combine scientific information with central planning as a new mode of educational policy making. At this time, their focus was a consequence of educational expansion, mainly on the design of a new structure for the lower secondary school level. Establishing planning offices was perceived as a prerequisite of school innovation. However, scientific information had to contend with political contradictions: democratising the school system on one hand, and conserving social status on the other. Burdened by this conflict, the planning of school innovation was only able to operate securely according to political rationality. Policy making in education has to take into account that scientific information is bound to different political rationales and operating procedures. 
T h e m a 